\title{
Bayesian Inference Based on Multiply Type-II Censored Sample from a General Class of Distributions
}

\author{
A.R. Shafay \\ Nature Science Department, Community College of Riyadh, King Saud University, \\ P.O. Box 28095, Riyadh 11437, Saudi Arabia \\ Department of Mathematics, Faculty of Science, Fayoum University, Fayoum, Egypt \\ a_shafay2013@yahoo.com \\ M.M. Mohie El-Din \\ Department of Mathematics, Faculty of Science, Al-Azhar University, Egypt \\ mmmmoheeldin@yahoo.com \\ Y. Abdel-Aty \\ Department of Mathematics, Faculty of Science, Al-Azhar University, Egypt \\ yahia1970@yahoo.com
}

Received 18 October 2016

Accepted 21 December 2017

\begin{abstract}
In this paper, we consider a general form for the underlying distribution and a general conjugate prior, and develop a general procedure for Bayesian estimation based on an observed multiply Type-II censored sample. The problem of predicting the order statistics from a future sample are also discussed from a Bayesian viewpoint. For the illustration of the developed results, the inverse Weibull distribution is used as example. Finally, two numerical examples are presented for illustrating all the inferential procedures developed here.
\end{abstract}

Keywords: Order statistics; Multiply Type-II censored sample; Bayesian prediction; Bayesian estimation; The inverse Weibull distribution; The inverse exponential distribution; The inverse Rayleigh distribution.

2000 Mathematics Subject Classification: 62G30, 62F15, 62N01

\section{Introduction}

In reliability analysis, experiments often get terminated before all units on test fail due to cost and time considerations. In such cases, failure information is available only on part of the sample, and only partial information on all units that had not failed. Such data are called censored data. There are several forms of censored data. One of the most common forms of censoring is Type-II right censoring which can be described as follows: Consider $n$ identical units under observation in a lifetesting experiment and suppose only the first $r \leq n$ failure times $X_{1: n}, \cdots, X_{r: n}$ are observed and the rest of the data are only known to be larger than $X_{r: n}$. A generalization of Type-II censoring scheme 
is multiple Type-II censoring scheme. Under this scheme, we observe only the $j_{1}^{t h}, j_{2}^{t h}, \cdots, j_{r}^{\text {th }}$ failure times $X_{j_{1}: n}, X_{j_{2}: n}, \ldots, X_{j_{r}: n}$, where $1 \leq j_{1}<j_{2}<\cdots<j_{r} \leq n$, and the rest of the data are not available. Particular applications of such censoring are found in reliability theory and survival analysis. Surveys regarding censored data can be found in Nelson [19], Balakrishnan and Cohen [6], Cohen [10], Balakrishnan and Aggarwala [5], and McCool [15]. For a survey on multiple Type-II censoring, one may refer to Kong [14].

Let $X_{1: n}<X_{2: n}<\cdots<X_{n: n}$ be the order statistics from a random sample of size $n$ from an absolutely continuous cumulative distribution function $(C D F) F(x) \equiv F(x \mid \theta)$ with probability density function $(P D F) f(x) \equiv f(x \mid \theta)$, where the parameter $\theta \in \Theta$ may be a real vector. These order statistics have been used in a wide range of problems, including robust statistical estimation, detection of outliers, characterization of probability distributions, goodness-of-fit tests, entropy estimation, analysis of censored samples, reliability analysis, quality control and strength of materials; for more details, see Arnold et al. [4], David and Nagaraja [11], Balakrishnan and Rao [7, 8], and the references contained therein. The joint density of multiply Type-II censored order statistics $\underline{X}=\left(X_{j_{1}: n}, X_{j_{2}: n}, \ldots, X_{j_{r}: n}\right)$ is given by

$$
f_{\underline{X}}(\underline{x})=\frac{n !}{\left(n-j_{r}\right) !}\left[1-F\left(x_{j_{r}}\right)\right]^{\left(n-j_{r}\right)} \prod_{i=1}^{r} \frac{1}{\left(j_{i}-j_{i-1}-1\right) !}\left[F\left(x_{j_{i}}\right)-F\left(x_{j_{i-1}}\right)\right]^{\left(j_{i}-j_{i-1}-1\right)} f\left(x_{j_{i}}\right),
$$

where $\underline{x}=\left(x_{j_{1}}, \ldots, x_{j_{r}}\right)$ is a vector of realizations and $j_{0}=0$.

We consider here the general inverse exponential form for the underlying distribution, suggested by Mohie El-Din et al. [16], that is described as follows; Motivated by the fact that the CDF can be written in the form

$$
F(x \mid \theta)=\exp [-\lambda(x ; \theta)],
$$

where $\lambda(x ; \theta)=-\ln F(x \mid \theta)$. Of course, some conditions need to be imposed so that $F(x \mid \theta)$ is a valid $C D F$. These conditions are: $\lambda(x ; \theta)$ is continuous, monotone decreasing and differentiable function, with $\lambda(x ; \theta) \rightarrow \infty$ as $x \rightarrow-\infty$ and $\lambda(x ; \theta) \rightarrow 0$ as $x \rightarrow \infty$. The PDF corresponding to (1.2) is given by

$$
f(x \mid \theta)=\lambda^{\prime}(x ; \theta) \exp [-\lambda(x ; \theta)],
$$

where $\lambda^{\prime}(x ; \theta)$ is the derivative of $\lambda(x ; \theta)$ with respect to $x$. With an appropriate choice of $\lambda(x ; \theta)$, several distributions that are used in reliability studies can be obtained as special cases such as the inverse exponential, inverse Rayleigh, inverse Weibull, inverse Pareto, negative exponential, negative Weibull, negative Pareto, negative power, Gumbel, exponentiated-Weibull, loglogistic, Burr X, inverse Burr XII and inverse paralogistic distributions.

In many practical problems, one may wish to use past data to predict an observation from a future sample from the same population. As in the case of estimation, a predictor can be either a point or an interval predictor. Several researchers have considered Bayesian prediction for future observations based on Type-II censored data; see Dunsmore [12], Nigm and Hamdy [22], Nigm [20, 21], AL-Hussaini and Jaheen [3], AL-Hussaini [2], and Raqab and Madi [23]. Bayesian prediction bounds for future observations based on multiply Type-II censored data have been discussed by several authors, including Abdel-Aty et al. [1], Schenk et al. [24], Mohie El-Din et al. [17], and Shafay et al. [25]. Recently, Mohie El-Din et al. [16] have considered the general inverse exponential form for the underlying distribution, given in (1.2), and a general conjugate prior and developed a 
general procedure for determining the two-sample Bayesian prediction for future lifetimes based on a right Type-II censored sample. In this paper, we discuss the same problem based on a multiply Type-II censored data which involves some additional complications.

The rest of this paper is organized as follows. In Section 2, we present the structure of the posterior distribution and derive the Bayesian estimation for the unknown parameters. In Section 3 , the problem of predicting the order statistics from a future sample is then discussed when the observed sample is a multiply Type-II censored sample from the same parent distribution. The results for the inverse Weibull distribution are presented in Section 4. Finally, in Section 5, we present some numerical results for illustrating all the inferential methods developed here.

\section{The Posterior Distribution and Bayesian Estimation}

In this section, we use the general inverse exponential form given in (1.2) and a general conjugate prior to develop general procedure for determining the Bayesian estimator of the unknown parameter $\theta$.

Upon using (1.2) and (1.3) in (1.1), we obtain the likelihood function of the multiply Type-II censored sample $\underline{X}=\left(X_{j_{1}: n}, X_{j_{2}: n}, \ldots, X_{j_{r}: n}\right)$ as

$$
L(\theta ; \underline{x}) \propto \sum_{k_{1}=1}^{j_{1}-j_{0}} \sum_{k_{r}=1}^{j_{r}-j_{r-1} n-j_{r}+1} \sum_{w=1}^{n} C_{k_{1}, \ldots, k_{r}, w} A(\theta ; \underline{x}) \exp [-B(\theta ; \underline{x})]
$$

where $C_{k_{1}, \ldots, k_{r}, w}=\left(\prod_{i=1}^{r} c_{k_{i}}\left(j_{i}-j_{i-1}\right)\right) c_{w}\left(n-j_{r}+1\right), c_{i}(j)=\frac{(-1)^{j-i}}{(i-1) !(j-i) !}$ for $i=1, \ldots, j$,

$$
A(\theta ; \underline{x})=\prod_{i=1}^{r}\left(-\lambda^{\prime}\left(x_{j_{i}} ; \theta\right)\right),
$$

and

$$
B(\theta ; \underline{x})=\left(n-j_{r}-w+1\right) \lambda\left(x_{j_{r}} ; \theta\right)+\sum_{i=1}^{r}\left(k_{i} \lambda\left(x_{j_{i}} ; \theta\right)+\left(j_{i}-j_{i-1}-k_{i}\right) \lambda\left(x_{j_{i-1}} ; \theta\right)\right) .
$$

From the Bayesian viewpoint, the unknown parameter is regarded as a realization of a random variable, which has some prior distribution. We consider here a general conjugate prior, suggested by AL-Hussaini [2], that is given by

$$
\pi(\theta ; \delta) \propto C(\theta ; \delta) \exp [-D(\theta ; \delta)],
$$

where $\theta \in \Theta$ is the vector of parameters of the distribution in (1.2) and $\delta$ is the vector of prior parameters. The prior family in (2.2) includes several priors used in the literature as special cases.

Upon combining (2.1) and (2.2), the posterior density function of $\theta$, given the multiply Type-II censored data, is obtained as

$$
\begin{aligned}
\pi^{*}(\theta \mid \underline{x}) & =L(\theta ; \underline{x}) \pi(\theta ; \delta) / \int_{\theta \in \Theta} L(\theta ; \underline{x}) \pi(\theta ; \delta) d \theta \\
& =I^{-1} \sum_{k_{1}=1}^{j_{1}-j_{0}} . \sum_{k_{r}=1}^{j_{r}-j_{r-1}} \sum_{w=1}^{n-j_{r}+1} C_{k_{1}, \ldots, k_{r}, w} \eta(\theta ; \underline{x}) \exp [-\zeta(\theta ; \underline{x})]
\end{aligned}
$$


where

$$
\eta(\theta ; \underline{x})=C(\theta ; \delta) A(\theta ; \underline{x}), \quad \zeta(\theta ; \underline{x})=B(\theta ; \underline{x})+D(\theta ; \delta),
$$

and

$$
I=\sum_{k_{1}=1}^{j_{1}-j_{0}} \cdot \sum_{k_{r}=1}^{j_{r}-j_{r-1} n-j_{r}+1} \sum_{w=1} C_{k_{1}, \ldots, k_{r}, w} \int_{\theta \in \Theta} \eta(\theta ; \underline{x}) \exp [-\zeta(\theta ; \underline{x})] d \theta
$$

The Bayesian estimator of $\theta$ under the squared error loss function is the mean of the posterior density function, given by

$$
\begin{aligned}
\widehat{\theta} & =E_{\pi^{*}(\theta \mid \underline{x})}[\theta] \\
& =I^{-1} \sum_{k_{1}=1}^{j_{1}-j_{0}} . \sum_{k_{r}=1}^{j_{r}-j_{r-1}} \sum_{w=1}^{n-j_{r}+1} C_{k_{1}, \ldots, k_{r}, w} \int_{\theta \in \Theta} \theta \eta(\theta ; \underline{x}) \exp [-\zeta(\theta ; \underline{x})] d \theta .
\end{aligned}
$$

\section{Bayesian Predication}

Let $Y_{1: m} \leq Y_{2: m} \leq \ldots \leq Y_{m: m}$ be the order statistics from a future random sample of size $m$ from the same population. We use here the general inverse exponential form given in (1.2) and the general conjugate prior given in (2.2) to develop a general procedure for deriving the point and interval predictions for $Y_{s: m}, 1 \leq s \leq m$, based on the observed multiply Type-II censored sample.

It is well known that the marginal density function of the $s^{\text {th }}$ order statistic from a sample of size $m$ from a continuous distribution with $C D F F(x)$ and $P D F f(x)$ is given, see Arnold et al. [4], by

$$
f_{Y_{s: m}}(y \mid \theta)=\frac{m !}{(s-1) !(m-s) !}[F(y)]^{s-1}[1-F(y)]^{m-s} f(y), \quad y>0 .
$$

Upon substituting (1.2) and (1.3) in (3.1), the marginal density function of $Y_{s: m}$ in (3.1) becomes

$$
f_{Y_{s: m}}(y \mid \theta)=\frac{m !}{(s-1) !} \sum_{q=1}^{m-s+1} c_{q}(m-s+1)\left(-\lambda^{\prime}(y ; \theta)\right) \exp [-(m-q+1) \lambda(y ; \theta)],
$$

where $c_{q}(m-s+1)=\frac{(-1)^{(m-s-q+1)}}{(q-1) !(m-s-q+1) !}$ for $q=1, \ldots, m-s+1$.

Upon combining (2.3) and (3.2), the Bayesian predictive density function of $Y_{s: m}$, given $\underline{X}=\underline{x}$, is then

$$
\begin{aligned}
f_{Y_{s: m}}^{*}(y \mid \underline{x})= & \int_{\theta \in \Theta} \pi^{*}(\theta \mid \underline{x}) f_{Y_{s: m}}(y \mid \theta) d \theta \\
= & \frac{m ! I^{-1}}{(s-1) !} \sum_{k_{1}=1}^{j_{1}-j_{0}} \cdot \sum_{k_{r}=1}^{j_{r}-j_{r-1} n-j_{r}+1} \sum_{w=1}^{n-s+1} \sum_{q=1}^{m} C_{k_{1}, \ldots, k_{r}, w} c_{q}(m-s+1) \\
& \quad \times \int_{\theta \in \Theta}\left(-\lambda^{\prime}(y ; \theta)\right) \eta(\theta ; \underline{x}) \exp [-(\zeta(\theta ; \underline{x})+(m-q+1) \lambda(y ; \theta))] d \theta .
\end{aligned}
$$


From (3.3), we simply obtain the predictive cumulative distribution function $F_{Y_{s: m}}^{*}(t \mid \underline{x})$, for $t \geq 0$, as

$$
\begin{aligned}
F_{Y_{s: m}}^{*}(t \mid \underline{x})= & \int_{0}^{t} f_{Y_{s: m}}^{*}(y \mid \underline{x}) d y \\
= & \frac{m ! I^{-1}}{(s-1) !} \sum_{k_{1}=1}^{j_{1}-j_{0}} \cdot \sum_{k_{r}=1}^{j_{r}-j_{r-1}} \sum_{w=1}^{n-j_{r}+1} \sum_{q=1}^{m-s+1} \frac{C_{k_{1}, \ldots, k_{r}, w} c_{q}(m-s+1)}{m-q+1} \\
& \times \int_{\theta \in \Theta} \eta(\theta ; \underline{x}) \exp [-\{\zeta(\theta ; \underline{x})+(m-q+1) \lambda(t ; \theta)\}] d \theta .
\end{aligned}
$$

The Bayesian point predictor of $Y_{s: m}$ under the squared error loss function is the mean of the predictive density, given by

$$
\widehat{Y}_{s: m}=\int_{0}^{\infty} y f_{Y_{s: m}^{*}}^{*}(y \mid \underline{x}) d y .
$$

The Bayesian predictive bounds of a two-sided equi-tailed $100(1-\gamma) \%$ interval for $Y_{s: m}, 1 \leq s \leq m$, can be obtained by solving the following two equations:

$$
F_{Y_{s: m}}^{*}\left(L_{Y_{s: m}} \mid \underline{x}\right)=\frac{\gamma}{2} \quad \text { and } \quad F_{Y_{s: m}}^{*}\left(U_{Y_{S: m}} \mid \mathbf{x}\right)=1-\frac{\gamma}{2},
$$

where $L_{Y_{S: m}}$ and $U_{Y_{S: m}}$ denote the lower and upper bounds, respectively.

Remark 3.1. In the case when the observed sample is right Type-II censored (i.e., $j_{i}=i, 1 \leq i \leq r$ ), the predictive density and cumulative distribution functions of $Y_{s: m}$ given in (3.3) and (3.4) reduce to expressions (2.13) and (2.14) of Mohie El-Din et al. [16], respectively.

\section{The Inverse Weibull Distribution}

Several distributions that are used in reliability studies can be obtained as special cases from the general inverse exponential form given in (1.2). We consider in this section the inverse Weibull distribution as illustrative example. The inverse Weibull model has been derived as a suitable model for describing the degradation phenomena of mechanical components, such as the dynamic components of diesel engines, see for example Murthy et al. [18]. The physical failure process given by Erto and Rapone [13] also leads to the inverse Weibull model. Erto and Rapone [13] showed that the inverse Weibull model provides a good fit to survival data such as the times to breakdown of an insulating fluid subject to the action of constant tension, see also Nelson [19]. Calabria and Pulcini [9] provided an interpretation of the inverse Weibull distribution in the context of a load-strength relationship for a component.

The distribution function of the inverse Weibull distribution is given by

$$
F(x \mid \theta)=\exp \left[-\alpha / x^{\beta}\right], \quad x>0,
$$

where $\alpha>0$ and $\beta>0$ are the scale and shape parameters, respectively, and so we have

$$
\lambda(x ; \alpha, \beta)=\alpha / x^{\beta} \text { and } \lambda^{\prime}(x ; \alpha, \beta)=-\alpha \beta / x^{\beta+1} .
$$

We provide here the Bayesian inference for the inverse Weibull distribution, when the scale parameter is unknown and the shape parameter is known. It is assumed that the scale parameter has a 
gamma prior distribution with the shape and scale parameters as $c$ and $d$, respectively and it has the density function

$$
\pi(\alpha ; \delta) \propto \alpha^{c-1} \exp [-d \alpha], \quad \alpha>0,
$$

where $c$ and $d$ can be chosen to suit the prior belief of the experimenter in terms of location and variability of the prior distribution. Moreover, Jeffrey's prior can be obtained as a special case of (4.2) by substituting $c=d=0$. So, we have

$$
C(\alpha ; \delta)=\alpha^{c-1} \text { and } D(\alpha ; \delta)=d \alpha,
$$

where $\delta=(c, d)$.

Hence, the posterior density function of $\alpha$, given the multiply Type-II censored data, become

$$
\begin{aligned}
\pi^{*}(\alpha \mid \underline{x})= & I^{-1} \sum_{k_{1}=1}^{j_{1}-j_{0}} . \sum_{k_{r}=1}^{j_{r}-j_{r-1}} \sum_{w=1}^{n-j_{r}+1} C_{k_{1}, \ldots, k_{r}, w} \alpha^{r+c-1} \\
& \times \exp \left[-\alpha\left(\frac{\left(n-j_{r}-w+1\right)}{x_{j_{r}}^{\beta}}+\frac{k_{1}}{x_{j_{1}}^{\beta}}+\sum_{i=2}^{r}\left(\frac{k_{i}}{x_{j_{i}}^{\beta}}+\frac{\left(j_{i}-j_{i-1}-k_{i}\right)}{x_{j_{i-1}}^{\beta}}\right)+d\right)\right],
\end{aligned}
$$

where

$I=\Gamma(r+c) \sum_{k_{1}=1}^{j_{1}-j_{0}} \cdot \cdot \sum_{k_{r}=1}^{j_{r}-j_{r-1}} \sum_{w=1}^{n-j_{r}+1} C_{k_{1}, \ldots, k_{r}, w}\left[\frac{\left(n-j_{r}-w+1\right)}{x_{j_{r}}^{\beta}}+\frac{k_{1}}{x_{j_{1}}^{\beta}}+\sum_{i=2}^{r}\left(\frac{k_{i}}{x_{j_{i}}^{\beta}}+\frac{\left(j_{i}-j_{i-1}-k_{i}\right)}{x_{j_{i-1}}^{\beta}}\right)+d\right]^{-(r+c)}$.

The Bayesian estimator of $\alpha$ is then given by

$$
\begin{aligned}
\widehat{\alpha}= & \Gamma(r+c+1) I^{-1} \sum_{k_{1}=1}^{j_{1}-j_{0}} \cdot \cdot \sum_{k_{r}=1}^{j_{r}-j_{r-1}} \sum_{w=1}^{n-j_{r}+1} C_{k_{1}, \ldots, k_{r}, w} \\
& \times\left[\frac{\left(n-j_{r}-w+1\right)}{x_{j_{r}}^{\beta}}+\frac{k_{1}}{x_{j_{1}}^{\beta}}+\sum_{i=2}^{r}\left(\frac{k_{i}}{x_{j_{i}}^{\beta}}+\frac{\left(j_{i}-j_{i_{-1}-k_{i}}\right)}{x_{j_{i-1}}^{\beta}}\right)+d\right]^{-(r+c+1)} .
\end{aligned}
$$

The predictive density and cumulative distribution functions of $Y_{s: m}$ are given, respectively, as

$$
\begin{aligned}
f_{Y_{s: m}}^{*}(y \mid \underline{x})= & \frac{m ! \Gamma(r+c+1) I^{-1}}{(s-1) !} \sum_{k_{1}=1}^{j_{1}-j_{0}} \cdot \sum_{k_{r}=1}^{j_{r}-j_{r-1}} \sum_{w=1}^{n-j_{r}+1} \sum_{q=1}^{m-s+1} \frac{\beta C_{k_{1}, \ldots, k_{r}, w} c_{q}(m-s+1)}{y^{\beta+1}} \\
& \times\left[\frac{\left(n-j_{r}-w+1\right)}{x_{j_{r}}^{\beta}}+\frac{k_{1}}{x_{j_{1}}^{\beta}}+\sum_{i=2}^{r}\left(\frac{k_{i}}{x_{j_{i}}^{\beta}}+\frac{\left(j_{i}-j_{i-1}-k_{i}\right)}{x_{j_{i-1}}^{\beta}}\right)+\frac{(m-q+1)}{y^{\beta}}+d\right]^{-(r+c+1)}
\end{aligned}
$$

and

$$
\begin{aligned}
F_{Y_{s: m}}^{*}(t \mid \underline{x})= & \frac{m ! \Gamma(r+c) I^{-1}}{(s-1) !} \sum_{k_{1}=1}^{j_{1}-j_{0}} . \sum_{k_{r}=1}^{j_{r}-j_{r-1}} \sum_{w=1}^{n-j_{r}+1} \sum_{q=1}^{m-s+1} \frac{C_{k_{1}, \ldots, k_{r}, w} c_{q}(m-s+1)}{m-q+1} \\
& \times\left[\frac{\left(n-j_{r}-w+1\right)}{x_{j_{r}}^{\beta}}+\frac{k_{1}}{x_{j_{1}}^{\beta}}+\sum_{i=2}^{r}\left(\frac{k_{i}}{x_{j_{i}}^{\beta}}+\frac{\left(j_{i}-j_{i-1}-k_{i}\right)}{x_{j_{i-1}}^{\beta}}\right)+\frac{(m-q+1)}{t^{\beta}}+d\right]^{-(r+c)} .
\end{aligned}
$$


The Bayesian point predictor of $Y_{s: m}$ under the squared error loss function is then obtained as the mean of the Bayesian predictive density function in (4.4), and this would require numerical integration. The Bayesian predictive bounds of a two-sided equi-tailed $100(1-\tau) \%$ interval for $Y_{s: m}$ can be obtained by solving the two equations in (3.6) where $F_{Y_{s: m}^{*}}^{*}(t \mid \underline{x})$ as in (4.5).

Remark 4.1. In the case when the observed sample is right Type-II censored (i.e., $j_{i}=i, 1 \leq i \leq r$ ), the posterior density function of $\alpha$ becomes

$$
\pi^{*}(\alpha \mid \underline{x})=I^{-1} \sum_{w=1}^{n-r+1} c_{w}(n-r+1) \alpha^{r+c-1} \exp \left[-\alpha\left(\frac{(n-r-w+1)}{x_{r}^{\beta}}+\sum_{i=1}^{r}\left(\frac{1}{x_{i}^{\beta}}\right)+d\right)\right],
$$

where

$$
I=\Gamma(r+c) \sum_{w=1}^{n-r+1} c_{w}(n-r+1)\left[\frac{(n-r-w+1)}{x_{r}^{\beta}}+\sum_{i=1}^{r}\left(\frac{1}{x_{i}^{\beta}}\right)+d\right]^{-(r+c)},
$$

and so the Bayesian estimator of $\alpha$ becomes

$$
\widehat{\alpha}=\Gamma(r+c+1) I^{-1} \sum_{w=1}^{n-r+1} c_{w}(n-r+1)\left[\frac{(n-r-w+1)}{x_{r}^{\beta}}+\sum_{i=1}^{r}\left(\frac{1}{x_{i}^{\beta}}\right)+d\right]^{-(r+c+1)} .
$$

The predictive density and cumulative distribution functions of $Y_{s: m}$ in this case become

$$
\begin{aligned}
f_{Y_{s: m}^{*}}^{*}(y \mid \underline{x})= & \frac{m ! \Gamma(r+c+1) I^{-1}}{(s-1) !} \sum_{w=1}^{n-r+1} \sum_{q=1}^{m-s+1} \frac{\beta c_{w}(n-r+1) c_{q}(m-s+1)}{y^{\beta+1}} \\
& \times\left[\frac{(n-r-w+1)}{x_{r}^{\beta}}+\sum_{i=1}^{r}\left(\frac{1}{x_{i}^{\beta}}\right)+\frac{(m-q+1)}{y^{\beta}}+d\right]^{-(r+c+1)}
\end{aligned}
$$

and

$$
\begin{aligned}
F_{Y_{s: m}}^{*}(t \mid \underline{x})= & \frac{m ! \Gamma(r+c) I^{-1}}{(s-1) !} \sum_{w=1}^{n-r+1} \sum_{q=1}^{m-s+1} \frac{c_{w}(n-r+1) c_{q}(m-s+1)}{m-q+1} \\
& \times\left[\frac{(n-r-w+1)}{x_{r}^{\beta}}+\sum_{i=1}^{r}\left(\frac{1}{x_{i}^{\beta}}\right)+\frac{(m-q+1)}{t^{\beta}}+d\right]^{-(r+c)} .
\end{aligned}
$$

Remark 4.2. The inverse Weibull distribution contains many of important special cases such as the inverse exponential and inverse Rayleigh distributions. The corresponding results of the inverse exponential and inverse Rayleigh distributions can be obtained by setting $\beta=1$ and 2, respectively.

\section{Numerical Results}

To illustrate the inferential procedures developed in the preceding sections, we present in this section a numerical study for the inverse exponential and inverse Rayleigh distributions as special cases of the inverse Weibull distribution when $\beta=1$ and $\beta=2$, respectively. 


\subsection{Numerical results for the inverse exponential distribution}

To illustrate the prediction results for the inverse exponential distribution, we generated order statistics from a sample of size $n=10$ from the inverse exponential distribution with $\alpha=5$. The generated order statistics are listed as follows: 1.31662, 3.04073, 5.25839, 5.39344, 5.64310, 5.88457, 13.72085, 22.43066, 29.19947, 1393.06058.

We then used these data to consider two different multiply Type-II censoring schemes:

(1) Scheme 1: $r=6$ with $j_{1}=1, j_{2}=3, j_{3}=5, j_{4}=6, j_{5}=7$ and $j_{6}=8$. Under this censoring scheme, we obtained the following data: 1.31662, 5.25839, 5.64310, 5.88457, 13.72085 and 22.43066;

(2) Scheme 2: $r=8$ with $j_{1}=1, j_{2}=3, j_{3}=4, j_{4}=5, j_{5}=6, j_{6}=7, j_{7}=9$ and $j_{8}=10$. Under this censoring scheme, we obtained the following data: 1.31662, 5.25839, 5.39344, 5.64310, 5.88457, 13.72085, 29.19947 and 1393.06058.

We assume these data to have come from the inverse exponential distribution, where the parameter $\alpha$ is unknown. Based on the above two multiply Type-II censoring schemes, we used the results presented earlier in Section 4 (when $\beta=1$ ) to compute the Bayesian estimate of $\alpha$. In addition, suppose we want to predict the order statistics from a future unobserved sample with size $m=10$. In this case, the point predictors and the 95\% two-sample Bayesian prediction intervals for the order statistics $Y_{s: 10}$, for $s=1, \ldots, 10$, are all obtained for three different choices of the prior, namely,

(1) Informative gamma prior 1 (GP 1) : $E(\alpha)=5$ and $\operatorname{Var}(\alpha)=2.5$. So, we have $c=10$ and $d=2$.

(2) Informative gamma prior (GP 2): $E(\alpha)=5$ and $\operatorname{Var}(\alpha)=5$. So, we have $c=5$ and $d=1$.

(3) Jeffreys' prior (JP): $\pi(\alpha) \propto 1 / \alpha$. So, we have $c=0$ and $d=0$.

The Bayesian estimates of $\alpha$ based on the three choices of the prior and the two multiply Type-II censoring schemes, are presented in Table 1. Table 2 shows the point predictors and the 95\% twosample Bayesian prediction intervals for the order statistics $Y_{s: 10}$, for $s=1, \ldots, 10$, based on the three choices of the prior and the two multiply Type-II censoring schemes.

Table 1. The Bayesian estimates of $\alpha$ based on the three choices of the prior and the two multiply Type-II censoring schemes.

\begin{tabular}{cccc}
\hline & GP 1 & GP 2 & JP \\
\hline Scheme 1 & 5.009 & 5.014 & 5.028 \\
Scheme 2 & 5.006 & 5.010 & 5.021 \\
\hline
\end{tabular}

\subsection{Numerical results of the inverse Rayleigh distribution}

To illustrate the prediction results for the inverse Rayleigh distribution, we generated order statistics from a sample of size $n=10$ from the inverse exponential distribution with $\alpha=5$. The generated order statistics are listed as follows: 1.35854, 1.80942, 2.04633, 2.08176, 2.34668, 2.94300, 4.81843, 5.26941, 8.16025, 59.60427.

We then used these data to consider two different multiply Type-II censoring schemes: 
Table 2. The Bayesian prediction for $Y_{s: 10}(s=1,2, \ldots, 10)$ based on the three choices of the prior and the two multiply Type-II censoring schemes.

\begin{tabular}{|c|c|c|c|c|c|c|c|c|c|c|}
\hline \multirow{2}{*}{$\begin{array}{c}\text { prior } \\
S\end{array}$} & & \multicolumn{3}{|c|}{ GP 1} & \multicolumn{3}{|c|}{ GP 2} & \multicolumn{3}{|c|}{ JP } \\
\hline & & $L_{Y_{s: 10}}$ & $U_{Y_{s: 10}}$ & $\widehat{Y}_{S: 10}$ & $L_{Y_{s: 10}}$ & $U_{Y_{s: 10}}$ & $\widehat{Y}_{S: 10}$ & $L_{Y_{s: 10}}$ & $U_{Y_{s: 10}}$ & $\widehat{Y}_{s: 10}$ \\
\hline \multirow[t]{2}{*}{1} & Scheme 1 & 0.721 & 4.619 & 2.094 & 0.686 & 4.737 & 2.462 & 0.620 & 4.971 & 3.017 \\
\hline & Scheme 2 & 0.722 & 4.616 & 1.986 & 0.687 & 4.733 & 2.153 & 0.621 & 4.964 & 2.681 \\
\hline \multirow[t]{2}{*}{2} & Scheme 1 & 1.153 & 6.715 & 3.045 & 1.092 & 6.889 & 3.538 & 0.980 & 7.233 & 4.916 \\
\hline & Scheme 2 & 1.154 & 6.710 & 2.952 & 1.093 & 6.883 & 3.242 & 0.983 & 7.223 & 4.142 \\
\hline \multirow[t]{2}{*}{3} & Scheme 1 & 1.563 & 9.230 & 4.172 & 1.479 & 9.460 & 4.627 & 1.326 & 9.919 & 5.788 \\
\hline & Scheme 2 & 1.565 & 9.224 & 4.089 & 1.480 & 9.453 & 4.383 & 1.329 & 9.905 & 5.094 \\
\hline \multirow[t]{2}{*}{4} & Scheme 1 & 2.012 & 12.611 & 5.490 & 1.906 & 12.907 & 5.837 & 1.710 & 13.494 & 6.980 \\
\hline & Scheme 2 & 2.014 & 12.603 & 5.243 & 1.909 & 12.895 & 5.462 & 1.714 & 13.477 & 6.103 \\
\hline \multirow[t]{2}{*}{5} & Scheme 1 & 2.543 & 17.563 & 7.287 & 2.411 & 17.940 & 7.798 & 2.170 & 18.695 & 9.358 \\
\hline & Scheme 2 & 2.546 & 17.555 & 7.074 & 2.416 & 17.927 & 7.359 & 2.176 & 18.671 & 8.869 \\
\hline \multirow[t]{2}{*}{6} & Scheme 1 & 3.209 & 25.583 & 9.996 & 3.067 & 26.067 & 10.350 & 2.755 & 27.043 & 11.907 \\
\hline & Scheme 2 & 3.210 & 25.569 & 9.526 & 3.104 & 26.040 & 10.042 & 2.758 & 27.010 & 11.005 \\
\hline \multirow[t]{2}{*}{7} & Scheme 1 & 4.101 & 40.475 & 14.542 & 3.908 & 41.115 & 14.991 & 3.548 & 42.423 & 16.007 \\
\hline & Scheme 2 & 4.109 & 40.450 & 14.282 & 3.915 & 41.083 & 14.638 & 3.554 & 42.368 & 15.215 \\
\hline \multirow[t]{2}{*}{8} & Scheme 1 & 5.400 & 75.022 & 22.780 & 5.165 & 75.931 & 23.081 & 4.721 & 77.798 & 24.457 \\
\hline & Scheme 2 & 5.439 & 74.978 & 22.361 & 5.171 & 75.769 & 22.584 & 4.726 & 77.699 & 23.762 \\
\hline \multirow[t]{2}{*}{9} & Scheme 1 & 7.563 & 199.92 & 46.646 & 7.269 & 201.38 & 47.159 & 6.705 & 204.44 & 48.724 \\
\hline & Scheme 2 & 7.669 & 199.01 & 45.127 & 7.274 & 201.22 & 46.827 & 6.714 & 204.19 & 47.951 \\
\hline \multirow[t]{2}{*}{10} & Scheme 1 & 12.340 & 1977.3 & 1071.8 & 11.95 & 1978.8 & 1074.5 & 11.172 & 1983.3 & 1085.7 \\
\hline & Scheme 2 & 12.433 & 1976.6 & 1069.1 & 11.99 & 1977.3 & 1072.9 & 11.188 & 1980.7 & 1080.3 \\
\hline
\end{tabular}

(1) Scheme 1: $r=6$ with $j_{1}=1, j_{2}=3, j_{3}=5, j_{4}=6, j_{5}=7$ and $j_{6}=8$. Under this censoring scheme, we obtained the following data: 1.35854, 2.04633, 2.34668, 2.94300, 4.81843 and 5.26941

(2) Scheme 2: $r=8$ with $j_{1}=1, j_{2}=3, j_{3}=4, j_{4}=5, j_{5}=6, j_{6}=7, j_{7}=9$ and $j_{8}=10$. Under this censoring scheme, we obtained the following data: 1.35854, 2.04633, 2.08176, 2.34668, 2.94300, 4.81843, 8.16025 and 59.60427.

We assume these data to have come from the inverse Rayleigh distribution, where the parameter $\alpha$ is unknown. Based on the above two multiply Type-II censoring schemes, we used the results presented earlier in Section 4 (when $\beta=2$ ) to compute the Bayesian estimate of $\alpha$. In addition, suppose we want to predict the order statistics from a future unobserved sample with size $m=10$. In this case, the point predictors and the 95\% two-sample Bayesian prediction intervals for the order statistics $Y_{s: 10}$, for $s=1, \ldots, 10$, are all obtained for three different choices of the hyper-parameters $c$ and $d$, namely,

(1) Informative gamma prior 1 (GP 1): $E(\alpha)=5$ and $\operatorname{Var}(\alpha)=2.5$. So, we have $c=10$ and $d=2$.

(2) Informative gamma prior 2 (GP 2): $E(\alpha)=5$ and $\operatorname{Var}(\alpha)=5$. So, we have $c=5$ and $d=1$.

(3) Jeffreys' prior (JP): $\pi(\alpha) \propto 1 / \alpha$. So, we have $c=0$ and $d=0$. 
The Bayesian estimates of $\alpha$ based on the three choices of the prior and the two multiply Type-II censoring schemes, are presented in Table 3. Table 4 shows the point predictors and the 95\% twosample Bayesian prediction intervals for the order statistics $Y_{s: 10}$, for $s=1, \ldots, 10$, based on the three choices of the prior and the two multiply Type-II censoring schemes.

Table 3. The Bayesian estimates of $\alpha$ based on the three choices of the prior and the two multiply Type-II censoring schemes.

\begin{tabular}{cccc}
\hline & GP 1 & GP 2 & JP \\
\hline Scheme 1 & 5.334 & 5.458 & 5.730 \\
Scheme 2 & 5.240 & 5.367 & 5.645 \\
\hline
\end{tabular}

Table 4. The Bayesian prediction for $Y_{s: 10}(s=1,2, \ldots, 10)$ based on the three choices of the prior and the two multiply Type-II censoring schemes.

\begin{tabular}{|c|c|c|c|c|c|c|c|c|c|c|}
\hline \multirow{2}{*}{$\begin{array}{c}\text { prior } \\
S\end{array}$} & & \multicolumn{3}{|c|}{ GP 1} & \multicolumn{3}{|c|}{ GP 2} & \multicolumn{3}{|c|}{ JP } \\
\hline & & $L_{Y_{S: 10}}$ & $U_{Y_{S: 10}}$ & $\widehat{Y}_{S: 10}$ & $L_{Y_{s: 10}}$ & $U_{Y_{s: 10}}$ & $\widehat{Y}_{S: 10}$ & $L_{Y_{S: 10}}$ & $U_{Y_{s: 10}}$ & $\widehat{Y}_{S: 10}$ \\
\hline \multirow[t]{2}{*}{1} & Scheme 1 & 0.877 & 2.217 & 1.415 & 0.865 & 2.269 & 1.735 & 0.843 & 2.377 & 1.945 \\
\hline & Scheme 2 & 0.878 & 2.216 & 1.392 & 0.866 & 2.268 & 1.682 & 0.844 & 2.376 & 1.874 \\
\hline \multirow[t]{2}{*}{2} & Scheme 1 & 1.109 & 2.672 & 1.713 & 1.092 & 2.736 & 1.941 & 1.060 & 2.867 & 2.105 \\
\hline & Scheme 2 & 1.110 & 2.270 & 1.699 & 1.093 & 2.734 & 1.887 & 1.062 & 2.865 & 2.002 \\
\hline \multirow[t]{2}{*}{3} & Scheme 1 & 1.291 & 3.133 & 2.000 & 1.271 & 3.206 & 2.402 & 1.233 & 3.357 & 2.784 \\
\hline & Scheme 2 & 1.292 & 3.131 & 1.979 & 1.273 & 3.203 & 2.038 & 1.235 & 3.354 & 2.572 \\
\hline \multirow[t]{2}{*}{4} & Scheme 1 & 1.465 & 3.663 & 2.291 & 1.442 & 3.745 & 2.696 & 1.401 & 3.917 & 2.903 \\
\hline & Scheme 2 & 1.466 & 3.660 & 2.287 & 1.444 & 3.740 & 2.473 & 1.402 & 3.913 & 2.711 \\
\hline \multirow[t]{2}{*}{5} & Scheme 1 & 1.647 & 4.323 & 2.632 & 1.623 & 4.416 & 3.091 & 1.577 & 4.609 & 3.334 \\
\hline & Scheme 2 & 1.648 & 4.322 & 2.622 & 1.624 & 4.412 & 2.806 & 1.580 & 4.605 & 3.049 \\
\hline \multirow[t]{2}{*}{6} & Scheme 1 & 1.850 & 5.217 & 3.060 & 1.825 & 5.323 & 3.547 & 1.777 & 5.545 & 3.908 \\
\hline & Scheme 2 & 1.852 & 5.210 & 3.019 & 1.828 & 5.320 & 3.176 & 1.780 & 5.542 & 3.631 \\
\hline \multirow[t]{2}{*}{7} & Scheme 1 & 2.091 & 6.563 & 3.646 & 2.065 & 6.686 & 4.007 & 2.017 & 6.947 & 4.817 \\
\hline & Scheme 2 & 2.093 & 6.561 & 3.573 & 2.067 & 6.682 & 3.824 & 2.020 & 6.941 & 4.230 \\
\hline \multirow[t]{2}{*}{8} & Scheme 1 & 2.410 & 8.935 & 4.498 & 2.374 & 9.088 & 5.626 & 2.326 & 9.410 & 6.715 \\
\hline & Scheme 2 & 2.411 & 8.931 & 4.466 & 2.377 & 9.081 & 5.415 & 2.329 & 9.402 & 5.988 \\
\hline \multirow[t]{2}{*}{9} & Scheme 1 & 2.840 & 14.588 & 6.130 & 2.816 & 14.802 & 7.474 & 2.771 & 15.258 & 8.970 \\
\hline & Scheme 2 & 2.842 & 14.497 & 6.072 & 2.822 & 14.714 & 7.278 & 2.775 & 15.218 & 8.002 \\
\hline \multirow[t]{2}{*}{10} & Scheme 1 & 3.627 & 45.883 & 14.136 & 3.610 & 46.410 & 16.857 & 3.577 & 47.542 & 18.545 \\
\hline & Scheme 2 & 3.639 & 44.912 & 12.612 & 3.623 & 45.645 & 14.849 & 3.582 & 46.604 & 17.013 \\
\hline
\end{tabular}

\subsection{Concluding Remarks}

From the results in Tables 1-4, the following points can be observed:

(1) The results obtained based on the multiply Type-II censoring scheme 2 (with $r=8$ ) is more precise than the corresponding ones based on the multiply Type-II censoring scheme 2 (with $r=6$ ), as we would expect; 
(2) A comparison of the results for the informative gamma priors with the corresponding ones for the Jeffreys' prior reveals that the former produce more precise results, as we would expect;

(3) The results obtained based on informative gamma prior 1 (with $\operatorname{Var}(\alpha)=2.5$ ) is more precise than the corresponding ones based on informative gamma prior $2($ with $\operatorname{Var}(\alpha)=5$ ), as we would expect;

(4) The width of the Bayesian prediction intervals decreases with increasing $\beta$.

\section{References}

[1] Y. Abdel-Aty, J. Franz and M.A.W. Mahmoud, Bayesian prediction based on generalized order statistics using multiply type II censored, Statistics 41 (2007) 495-504.

[2] E.K. AL-Hussaini, Predicting observables from a general class of distributions, J. Statist. Plann. Inf. 79 (1999) 79-91.

[3] E.K. AL-Hussaini and Z.F. Jaheen, Bayesian prediction bounds for the Burr type XII model, Comm. Statist. Theor. Meth. 24 (1995) 1829-1842.

[4] B.C. Arnold, N. Balakrishnan and H.N. Nagaraja, A First Course in Order Statistics, (John Wiley \& Sons, New York, 1992).

[5] N. Balakrishnan and R. Aggarwala, Progressive Censoring: Theory, Methods and Applications, (Birkhäuser, Boston, 2000).

[6] N. Balakrishnan and A.C. Cohen, Order Statistics and Inference: Estimation Methods, (Academic Press, Boston, 1991).

[7] N. Balakrishnan and C.R. Rao, Order Statistics: Theory and Methods, Handbook of statistics - vol. 16, (North-Holland, Amsterdam, $1998^{a}$ ).

[8] N. Balakrishnan and C.R. Rao, Order Statistics: Theory and Methods, Handbook of statistics - vol. 17, (North-Holland, Amsterdam, $1998^{b}$ ).

[9] R. Calabria and G. Pulcini, Bayesian 2-sample prediction for the inverse Weibull distribution, Comm. Statist. Theor. Meth. 23 (1994) 1811-1824.

[10] A.C. Cohen, Truncated and Censored Samples: Theory and Applications, (Marcel Dekker, New York, 1991).

[11] H.A. David and H.N. Nagaraja, Order Statistics, Third edition, (John Wiley \& Sons, Hoboken, New Jersey, 2003).

[12] I.R. Dunsmore, The Bayesian predictive distribution in life testing models, Technometrics 16 (1974) $455-460$

[13] P. Erto and M. Rapone, Non-informative and practical Bayesian confidence bounds for reliable life in the Weibull model, Reliab. Eng. 7 (1984) 181-191.

[14] F. Kong, Parameter estimation under multiply Type-II censoring, In: N. Balakrishnan and C.R. Rao (Eds.), Handbook of Statistics - Order Statistics: Applications, Vol. 17, pp. 315-335, (Elsevier, Amsterdam, 1998).

[15] J.I. McCool, Censored data. In: S. Kotz, N. Balakrishnan, C.B. Read and B. Vidakovic (Eds.), Encyclopedia of Statistical Sciences, Second ed., (John Wiley \& Sons, Hoboken, New Jersey,2006).

[16] M.M. Mohie El-Din, Y. Abdel-Aty and A.R. Shafay, Two sample Bayesian prediction intervals for order statistics based on the inverse exponential-type distributions using right censored sample, J. Egyp. Math. Society 19 (2011) 102-105.

[17] M.M. Mohie El-Din and A.R. Shafay, Two-Sample Bayesian Prediction Intervals of Generalized Order Statistics Based on Multiply Type II Censored Data, Comm. Statist. Theor. Meth. 41 (2012) 381-392.

[18] D.N.P. Murthy, M. Xie and R. Jiang, Weibull Models, (Wiley, New York, 2004).

[19] W. Nelson, Applied Lifetime Data Analysis, (Wiley, New York, 1982).

[20] A.M. Nigm, Prediction bounds for the Burr model, Comm. Statist. Theor. Meth. 17 (1988) 289-297.

[21] A.M. Nigm, An informative Bayesian prediction for the Weibull lifetime distribution, Comm. Statist. Theor. Meth. 18 (1989) 897-911. 
[22] A.M. Nigm and H.I. Hamdy, Bayesian prediction bounds for the Pareto lifetime model, Comm. Statist. Theor. Meth. 16 (1987) 1761-1772.

[23] M.Z. Raqab and M.T. Madi, Bayesian inference for the generalized exponential distribution, J. Statist. Comput. Simul. 69 (2005) 109-124.

[24] N. Schenk, M. Burkschat, E. Cramer and U. Kamps, Bayesian estimation and prediction with multiply Type-II censored samples of sequential order statistics from one- and two-parameter exponential distributions, J. Statist. Plann. Inf. 141 (2011) 1575-1587.

[25] A.R. Shafay, N. Balakrishnan and K.S. Sultan, Two-sample Bayesian prediction for sequential order statistics from exponential distribution based on multiply Type-II censored samples, J. Statist. Comput. Simul. 84 (2014) 526-544. 